\title{
Immunization Against Hepatitis A Virus and Hepatitis B Virus in Patients with Chronic Liver Disease: Are We Doing a Good Job?
}

\author{
Rutaba Tajammal ${ }^{1}$, Ijlal Akbar Ali ${ }^{2}$, Taseen Syed ${ }^{1}$, Salman Nusrat ${ }^{3}$ \\ 1. Internal Medicine, University of Oklahoma Health Sciences Center, Oklahoma City, USA 2. Section of \\ Digestive Diseases and Nutrition, University of Oklahoma Health Sciences Center, Oklahoma City, USA 3. \\ Gasteroenterology, University of Oklahoma Health Sciences Center, Oklahoma City, USA
}

$\square$ Corresponding author: Rutaba Tajammal, rutabasultan@hotmail.com Disclosures can be found in Additional Information at the end of the article

\section{Abstract}

Introduction: In the era of highly effective vaccines for Hepatitis A Virus (HAV) and Hepatitis B Virus (HBV), acute viral hepatitis in patients with a chronic liver disease remains a public health concern. Vaccination for HAV and HBV is endorsed by all liver society guidelines. The aim of our study was to determine the rates of immunization in an internal medicine resident clinic.

Methods: We identified patients with a chronic liver disease seen at the University of Oklahoma Internal Medicine resident clinic between June 2014 and May 2015. ICD-9 code 571 was used to identify patients with a chronic liver disease. Vaccination records and patient data were reviewed.

Results: A total of 141 patients with a chronic liver disease (mean age 54.1 years, 56\% males) were identified. Almost half of the patients (47.5\%) were also being seen in the gastroenterology clinic. During the internal medicine resident clinic visit, vaccination against HAV and HBV was addressed for $50 \%$ and $46 \%$ of the patients, respectively. Patients being seen by senior residents were more likely to be immunized against HAV (OR 2.7, p=0.009) and HBV (OR 2.1, p=0.03). Patients followed in the GI clinic were more likely to be immunized against HAV (OR 2.1, p= 0.02) and HBV (OR 2.0, p=0.02). The gender of the treating physician and etiology had no impact on vaccination rates.

Discussion: Immunization rates for HAV and HBV remain subpar despite clear guidelines for patients with a chronic liver disease. This provides an important avenue for improvement. Different strategies, including resident education, developing vaccination protocols, and referral to the gastroenterology clinic, are likely to improve vaccination status for patients with chronic liver diseases.

Categories: Internal Medicine, Preventive Medicine, Gastroenterology

Keywords: hepatitis b, vaccination, hepatitis a, chronic liver disease, primary care, cirrhosis

\section{Introduction}

In the era of highly effective vaccines for Hepatitis A Virus (HAV) and Hepatitis B Virus (HBV), acute viral hepatitis remains a public health concern [1]. As compared to the general

\section{How to cite this article}

Tajammal R, Ali I, Syed T, et al. (April 24, 2018) Immunization Against Hepatitis A Virus and Hepatitis B Virus in Patients with Chronic Liver Disease: Are We Doing a Good Job?. Cureus 10(4): e2528. DOI 10.7759/cureus. 2528 
population, patients with chronic liver diseases are predisposed to severe hepatitis and possible liver failure if they contract an HAV or HBV infection [2-4]. As the prevalence of non-alcoholic fatty liver disease (NAFLD) and cirrhosis rises in the hospitalized patient population, patients with an acute hepatitis infection are likely to have a prolonged and complicated hospital course [2-4]. Patients with a pre-existing liver disease are also more likely to develop acute hepatitis-related complications, including encephalopathy and/or ascites [5-6]. Studies have shown that HAV and HBV vaccination is safe and effective in patients with chronic liver diseases [2]. The Centers for Disease Control and Prevention, the National Institutes of Health, the Veteran's Health Administration, and the American Liver Foundation are among the organizations endorsing vaccination of patients with chronic liver diseases [7].

The aim of our study was to determine the rates of HAV and HBV vaccination among patients with chronic liver diseases presenting to the internal medicine resident clinic at the University of Oklahoma Health Sciences Center.

\section{Materials And Methods}

Patients with chronic liver diseases seen at University of Oklahoma Internal Medicine resident clinic between June 2014 and May 2015 were identified using ICD-9 code 571. Demographic data, the etiology of the liver disease, clinic information, and treating physician information were extracted. Vaccination records were also reviewed. Patients were considered immunized if they had documented immunoglobulin G (IgG) HAV antibody (Ab) and IgG HBV surface antibody (HBV sAb) or they had documented vaccination administered in the clinic.

\section{Results}

A total of 141 patients with a mean age of 54.1 years and $56 \%$ males were identified. Hepatitis C Virus (HCV) was the most common etiology (48\%) followed by alcoholic liver disease in $21 \%$ and NAFLD in $20 \%$. Almost half of the patients (47.5\%) were also cared for in the gastroenterology clinic. During the internal medicine resident clinic visit, vaccination against $\mathrm{HAV}$ and HBV was addressed for $50 \%$ and $46 \%$ of the patients, respectively. IgG Ab against HAV was present in $47.5 \%$ patients, and $2.5 \%$ were identified as being immunized by two or more documented vaccinations against HAV. HBV sAb was present in $40 \%$, and $6 \%$ were identified as being immunized by three documented vaccinations against HBV. Patients being seen by a senior medicine resident (PGY2 to PGY4) were more likely to be immunized against HAV (odds ratio (OR) 2.7, confidence interval (CI) 1.2-5.8, $\mathrm{p}=0.009$ ) and HBV (OR 2.1, CI 1.05-4.4, $\mathrm{p}=0.03$ ). Patients who were also being followed in the GI clinic were more likely to be immunized against HAV (OR 2.1, CI 1.3-3.9, p= 0.02) and HBV (OR 2.0, CI 1.1-3.7, p=0.02). The gender of the treating physician and the etiology of the chronic liver disease had no impact on documenting vaccination. The impact of different factors on vaccination rates for patients with chronic liver diseases is presented in Table 1. 


\section{Cureus}

\begin{tabular}{|c|c|c|}
\hline & HAV vaccination & HBV vaccination \\
\hline Senior Residents (PGY2 to PGY4) & OR 2.7, Cl 1.2-5.8, p=0.009 & OR $2.1, \mathrm{Cl} 1.05-4.4, \mathrm{p}=0.03$ \\
\hline GI Clinic Follow-Up & OR 2.1, Cl 1.3-3.9, p= 0.02 & OR 2.0, Cl 1.1-3.7, p=0.02 \\
\hline Etiology of Chronic Liver Disease (HCV) & OR $1.2, \mathrm{Cl} 0.7-1.4, p=0.7$ & OR $1.1, \mathrm{Cl} 0.7-1.3, p=0.8$ \\
\hline Gender (Male) & OR $1.1, \mathrm{Cl} 0.5-1.3, p=0.6$ & OR $1.2, \mathrm{Cl} 0.4-1.9, \mathrm{p}=0.8$ \\
\hline
\end{tabular}

\section{TABLE 1: Impact of Different Factors on Vaccination Rates for Patients with Chronic Liver Diseases}

OR: odds ratio; $\mathrm{Cl}$ : confidence interval

\section{Discussion}

Preventive care is the best and the most cost-effective way to improve patient outcomes and prevent untoward complications [8]. There are limited studies looking at the vaccination rates for patients with chronic liver diseases. Although vaccination rates for HAV and HBV in patients with chronic liver diseases have been variable, in most cases, they remain subpar [9]. One study showed that vaccination rates are lower in the primary care clinic as compared to the specialty clinic [10].

The resident clinic at a university hospital is an important avenue to provide quality care to patients with limited access to healthcare. Most patients coming to the resident clinic fall in the low-income category and are uninsured or underinsured [11]. This means that the resident clinic visit may be the only place for them to seek comprehensive healthcare. Most patients don't have access to specialty care and, as seen in our study, less than half of the patients were being seen in the gastroenterology specialty clinic.

There can be multiple reasons for low vaccination rates in the resident clinic, including busy workload and lack of knowledge of current guidelines. Studies have highlighted variations in quantifiable outcomes and adherence to well-established guidelines among primary care physicians as well as specialists [10-12]. There are several ways to overcome this issue, including physician education [13], scheduled reminders, and recall systems [14-15]. An electronic health system is also an important tool that can be used to improve vaccination coverage by adding automatic reminders for patients with chronic liver diseases [16].

There are several limitations of this study. This was a retrospective study that was performed at a single center. We also did not record the severity of liver disease and assumed that the patient was not vaccinated if there were no documented vaccination on the chart. It is possible that those patients might have received vaccinations elsewhere.

The abstract of our research study was presented as a poster at the 2015 American College of Gastroenterology Annual Scientific Meeting by Iftikhar O, Mahmood S, Ali IA, et al.

\section{Conclusions}

In summary, despite clear guidelines for the immunization of patients with chronic liver 
diseases, a significant number of patients are not adequately vaccinated against HAV and HBV, putting them at increased risk of contracting these infections. Educating resident physicians, developing vaccination protocols, and referral to the Gastroenterology clinic are likely to improve vaccination status for patients with chronic liver diseases.

\section{Additional Information \\ Disclosures}

Human subjects: Consent was obtained by all participants in this study. Office of Human Research Participant Protection IRB OUHSC issued approval N/A. The study was conducted as a quality improvement project and was deemed exempt from IRB approval. Animal subjects: All authors have confirmed that this study did not involve animal subjects or tissue. Conflicts of interest: In compliance with the ICMJE uniform disclosure form, all authors declare the following: Payment/services info: All authors have declared that no financial support was received from any organization for the submitted work. Financial relationships: All authors have declared that they have no financial relationships at present or within the previous three years with any organizations that might have an interest in the submitted work. Other relationships: All authors have declared that there are no other relationships or activities that could appear to have influenced the submitted work.

\section{References}

1. Koenig A, Stepanova M, Felix S, Kalwaney S, Clement S, Younossi ZM: Vaccination against hepatitis A and B in patients with chronic liver disease and type 2 diabetes: has anything changed?. Liver Int. 2016, 36:1096-1100. 10.1111/liv.13164

2. Keeffe EB: Acute hepatitis A and B in patients with chronic liver disease: prevention through vaccination. Am J Med. 2005, 118:21-27. 10.1016/j.amjmed.2005.07.013

3. Collier MG, Tong X, Xu F: Hepatitis A hospitalizations in the United States, 2002-2011 . Hepatology. 2015, 61:481-485. 10.1002/hep.27537

4. Sagnelli E, Coppola N, Messina V, et al.: HBV superinfection in hepatitis C virus chronic carriers, viral interaction, and clinical course. Hepatology. 2002, 36:1285-1291. 10.1053/jhep.2002.36509

5. Singh KK, Panda SK, Acharya SK: Patients with diabetes mellitus are prone to develop severe hepatitis and liver failure due to hepatitis virus infection. J Clin Exp Hepatol. 2013, 3:275-280. 10.1016/j.jceh.2013.11.003

6. Sarin SK, Kumar A, Almeida JA, et al.: Acute-on-chronic liver failure: consensus recommendations of the Asian Pacific Association for the Study of the Liver (APASL). Hepatol Int. 2009, 3:269. 10.1007/s12072-008-9106-X

7. Reiss G, Keeffe E: Hepatitis vaccination in patients with chronic liver disease . Aliment Pharmacol Ther. 2004, 19:715-727. 10.1111/j.1365-2036.2004.01906.x

8. Woolf SH: The power of prevention and what it requires . Jama. 2008, 299:2437-2439. 10.1001/jama.299.20.2437

9. Thudi K, Yadav D, Sweeney K, Behari J: Physicians infrequently adhere to hepatitis vaccination guidelines for chronic liver disease. PloS One. 2013, 8:71124. 10.1371/journal.pone.0071124

10. Jacobs RJ, Meyerhoff AS, Saab S: Immunization needs of chronic liver disease patients seen in primary care versus specialist settings. Dig Dis Sci. 2005, 50:1525-1531. 10.1007/s10620-0052873-5

11. Holmboe ES, Prince L, Green M: Teaching and improving quality of care in a primary care internal medicine residency clinic. Acad Med. 2005, 80:571-577.

12. Barclay RL, Vicari JJ, Doughty AS, Johanson JF, Greenlaw RL: Colonoscopic withdrawal times and adenoma detection during screening colonoscopy. N Engl J Med. 2006, 355:2533-2541. 10.1056/NEJMoa055498

13. Davis DA, Thomson MA, Oxman AD, Haynes RB: Changing physician performance: a systematic review of the effect of continuing medical education strategies. Jama. 1995, 274:700-705. 10.1001/jama.1995.03530090032018 


\section{Cureus}

14. Jacobson Vann JC, Szilagyi P: Patient reminder and recall systems to improve immunization rates. Cochrane Database Syst Rev. 2005, 3:CD003941. 10.1002/14651858.CD003941.pub2

15. Tape TG, Campbell JR: Computerized medical records and preventive health care: success depends on many factors. Am J Med. 1993, 94:619-625. 10.1016/0002-9343(93)90214-A

16. Au L, Oster A, Yeh G, Magno J, Paek H: Utilizing an electronic health record system to improve vaccination coverage in children. Appl Clin Inform. 2010, 1:221. 10.4338/ACI-2009-12-CR0028 